9 Tablan $O C$, Anderson $L J$, Arden NH, et al. Guideline for prevention of nosocomial pneumonia. Part I. Issues on prevention of nosocomial pneumonia - 1994. Part II. Recommendations for prevention of nosocomial pneumonia. Am J Infect Control 1994; 22: 247-92.

10 Subcommittee on Infection Control Policy. Prevention of nosocomial infections in patients. In: Recommendations for Infection Control for the Practice of Anesthesiology, Park Ridge: American Society of Anesthesiologists, 1992; 2-16.

11 Favero MS. Principles of sterilization and disinfection. Anesth Clin N Am 1989; 7: 941-50.

12 Garibaldi $R A$, Britt $M R$, Webster C, Pace NL. Failure of bacterial filters to reduce the incidence of pneumonia after inhalation anesthesia. Anesthesiology 1981; 54: 364-8.

13 Campbell BA, Wells GA, Palmer WN, Martin DL. Reuse of disposable medical devices in Canadian hospitals. Am J Infect Control 1987; 15: 196-200.

14 Greene $V W$. Reuse of disposable medical devices: historical and current aspects. Infection Control 1986; 7: 508-13.

\section{Management of postoperative pain and emesis}

\author{
Paul F. White PhD MD FANZCA \\ Department of Anesthesiology and Pain Management, \\ University of Texas Southwestern Medical Center at \\ Dallas.
}

"Slapping the patient on the face and telling him or her that it's all over is a complete inversion of the truth. As far as the patient is concerned, it is often just the beginning." Although the currently available armamentarium of analgesic and antiemetic drugs is impressive, management of acute postoperative pain, as well as nausea and vomiting, poses some unique challenges following ambulatory surgery. The increasing number and complexity of operations being performed on an outpatient basis present the practitioners of ambulatory anaesthesia with many unique challenges. Outpatients undergoing day-case procedures require an analgesic technique that is effective, has minimal side effects, is intrinsically safe, and can be easily managed away from the hospital or surgery centre. Intractable nausea and vomiting not only contributes to dehydration in the early recovery period and delays the discharging of patients from the ambu- latory unit, but also leads to unanticipated hospital admissions.

The control of postoperative pain and emesis is the most important factor in determining when a patient can be safely discharged from an outpatient facility. Since inadequately treated pain and emesis are among the most common problems after ambulatory surgery, the ability to provide adequate pain relief without exacerbating postoperative nausea and vomiting (PONV) remains one of the major challenges for providers of outpatient anaesthesia and surgery. Although perioperative analgesia has traditionally been provided by opioid analgesics, aggressive use of opioids can be associated with sedation and an increased incidence of PONV, which, in turn, contributes to a delayed discharge from the day-care facility. In order to minimize these opioid-related adverse effects, "balanced" analgesia techniques involving the use of opioid and non-opioid analgesic drugs (local anaesthetics and non-steroidal anti-inflammatory drugs (NSAIDs) are becoming increasingly popular. ${ }^{2}$

\section{Local anaesthetic techniques}

Peripheral nerve blocks and wound infiltration with local anaesthetics are becoming increasingly popular adjuvants to general anaesthesia because they can provide considerable intraoperative and postoperative analgesia. These techniques decrease the incidence of pain and reduce the requirements for narcotic analgesics in the perioperative period. Effective pain relief in the early postoperative period provides for rapid and smooth recovery, enabling earlier ambulation and discharge from the ambulatory surgery unit. The use of local anaesthetic techniques for postoperative pain control can also decrease the incidence of PONV and, thereby, potentially lower the incidence of unanticipated hospital admission after ambulatory surgery. For example, blockade of the ilioinguinal and iliohypogastric nerves with bupivacaine $0.25-0.5 \%$ decreases the anaesthetic and analgesic requirements in children and adults undergoing inguinal herniorraphy. Subcutaneous ring block of the penis with bupivacaine $0.25 \%$ provides effective analgesia after circumcision. Similarly, infiltration of the mesosalpinx in the area of the Yoon ring placement with $0.5 \%$ bupivacaine decreases the postoperative pain and cramping after laparoscopic tubal ligation. Pain after knee surgery can be decreased by administering a femoral nerve block alone or in combination with intraarticular local anaesthesia.

While subcutaneous infiltration of the operative site with local anaesthetics remains a popular technique for decreasing postoperative opioid analgesic requirement, other simplified local anaesthetic techniques have been described in the anaesthesia literature. For example, topical analgesia with a lidocaine aerosol was found to be 
highly effective in decreasing pain, as well as the opioid analgesic requirement after inguinal herniorraphy. Instillation of bupivacaine $0.5 \%$ into the hernia wound prior to fascial closure provided similar postoperative pain relief to that achieved with an ilioinguinal-iliohypogastric nerve block in children. The simple application of topical lidocaine jelly, lidocaine ointment, or lidocaine spray has been shown to be as effective as peripheral nerve blocks and parenteral opioids in providing pain relief after outpatient circumcision. Intraperitoneal administration of local anaesthetics $(80 \mathrm{ml}$ lidocaine $0.5 \%$ and bupivacaine $0.125 \%$ with epinephrine) during laparoscopy was found to be an effective method for reducing the intensity of postoperative scapular pain. Local anaesthetics (e.g., bupivacaine $0.5 \%$ ) instilled into the knee and shoulder joints provide effective analgesia during arthroscopic surgery and facilitate early recovery. In recent years, increasing evidence has been obtained to suggest that intraarticular morphine, either alone or in combination with bupivacaine, can also produce effective and long-lasting analgesia following arthroscopic surgery.

\section{Non-steroidal anti-inflammatory drugs}

Oral non-steroidal anti-inflammatory drugs (NSAIDs) have long been used in medicine for their antiinflammatory, antipyretic and analgesic properties. With the introduction of parenteral preparations of NSAIDs (e.g., ketorolac), more widespread use of these drugs has been reported in the management of postoperative pain. Since the NSAIDs were alleged to have analgesic properties comparable with opioid compounds but without opioid-related side effects, anaesthetists often administered these drugs as adjuvants during and immediately after ambulatory surgery. ${ }^{3}$ When ketorolac was administered as an adjuvant to propofol- $\mathrm{N}_{2} \mathrm{O}$ anaesthesia, it was associated with improved postoperative analgesia and patient comfort compared with fentanyl. Furthermore, the administration of ketorolac as an alternative to fentanyl for augmentation of local anaesthesia during monitored anaesthesia care resulted in considerably less postoperative pruritus, nausea and vomiting.

Due to the weaker intrinsic analgesic properties of the NSAIDs (vs opioid analgesics and local anaesthetics), these compounds have limited use in the management of acute intraoperative pain. However, despite the seemingly conflicting data in the literature, the NSAIDs are useful adjuvants in the management of postoperative pain in the ambulatory setting. Concerns regarding the potentially adverse effects of NSAIDs on platelet and renal function are less with short-term administration after ambulatory surgery. Importantly, it appears that the clinical efficacy and safety of this group of analgesic drugs depends upon the timing and route of administration, as well as the surgical procedure itself. Finally, since the NSAIDs may be associated with less postoperative nausea, vomiting and respiratory depression than the opioid analgesic drugs, their use in outpatient anaesthesia will contribute not only to a shorter postoperative recovery period, but may also lead to improved patient comfort and safety.

\section{Non-pharmacological techniques}

Transcutaneous electric nerve stimulation (TENS) or acupuncture-like transcutaneous electrical nerve stimulation (ALTENS), as well as percutaneous electrical nerve stimulation (PENS), have been utilized in the treatment of both acute and chronic pain in the ambulatory setting. Given the inherent side effects produced by both opioid and non-opioid analgesics, as well as the local anaesthetics, it is not surprising that nonpharmacological approaches to managing acute postoperative pain are increasingly popular in the outpatient setting. The mechanisms by which TENS, ALTENS, and PENS exert their analgesic action have not been completely elucidated. However, possible mechanisms include: (1) stimulation of descending pain inhibitory pathways, (2) an inhibition of substance-P release in central nervous system (CNS) structures, and (3) the release of endogenous opioid substances within the CNS.

\section{Postoperative emesis}

Nausea, retching and vomiting are among the most common postoperative complaints which occur after general, regional and local anaesthesia. ${ }^{4}$ Although much less problematic in patients receiving local anaesthesia as part of a monitored anaesthetic care technique, the incidence of postoperative emesis in recent large studies has been reported to be in the $20-30 \%$ range, which is consistently lower than the $75-80 \%$ incidence reported during the "ether" era. Nevertheless, persistent nausea and vomiting may result in dehydration, electrolyte imbalance, and delayed discharge after outpatient surgery. Persistent retching or vomiting can cause tension on suture lines, venous hypertension and increased bleeding under skin flaps, as well as exposing the patient to an increased risk of pulmonary aspiration of vomitus (if the airway reflexes are depressed from the residual effects of anaesthetic and analgesic drugs). Although frequently described as a "minor" postoperative complication, the incidence of severe (intractable) nausea and vomiting has been reported to be 1 in $1000(0.1 \%)$. Factors affecting postoperative nausea and emesis include the patient's demographic characteristics, the nature of the underlying disease for which the surgery is being performed, the type of operation, and the anaesthetic drugs and techniques.

In a recent editorial, Kapur described perioperative 
nausea and vomiting as "the big little problem following ambulatory surgery." Although the morbidity associated with nausea is relatively low in healthy outpatients, it should not be considered an unavoidable part of the perioperative experience. While there has been little change in the incidence of postoperative emesis since the introduction of halothane, newer anaesthetic drugs (e.g., propofol) appear to have contributed to the recent decline in the incidence of emesis. ${ }^{6}$ Factors associated with an increased risk of postoperative emesis include age, sex (menses), obesity, previous history of motion sickness or postoperative vomiting, anxiety, gastroparesis, type and duration of the surgical procedure (e.g., laparoscopy, strabismus, middle ear procedures, endosinus surgery). While anaesthetists have little control over the surgical factors, they do have control over many other factors that influence postoperative emesis (e.g., preanaesthetic medication, anaesthetic drugs and techniques, hydration status, and postoperative pain management). Although "routine" antiemetic prophylaxis is unjustified, patients at high risk for postoperative emesis should receive special consideration with respect to the prophylactic use of antiemetic drugs.

Ondansetron, $4 \mathrm{mg} \dot{i}$, is highly effective in both the prevention and treatment of PONV. Importantly, the use of this new 5-HT ${ }_{3}$ antagonist is associated with an excellent side effect profile. Administration of "minimally effective" doses of droperidol $(0.625 \mathrm{mg} i$ iv) will minimize the side effects (e.g., sedation, restlessness, dysphoria) associated with this otherwise useful antiemetic. Potent nonopioid analgesics (e.g., local anaesthetics, ketorolac) can be used to control postoperative pain while avoiding opioid-related side effects. Gentle handling and movement of the patient in the immediate postoperative period is also essential. If prolonged (or recurrent) emesis occurs after ambulatory surgery, aggressive intravenous hydration and pain management are important components of the therapeutic regimen along with the use of antiemetic drugs. If one antiemetic does not appear to be effective, another drug with a different site of action should be considered (e.g., dopamine antagonist vs $5-\mathrm{HT}_{3}$ antagonist). With the availability of new antiserotonin drugs (e.g., ondansetron, granisetron), the incidence of recurrent emesis should be further decreased in the future. Most outpatients, as well as those involved in their postoperative care, look forward to a time when the routine offering of an emesis basin after ambulatory surgery becomes an historical practice.

\section{Summary}

As more extensive and painful surgical procedures (e.g., laparoscopic cholecystectomy, laminectomy, knee and shoulder reconstruction, hysterectomy) are being per- formed on an outpatient basis, the availability of sophisticated postoperative analgesic regimens are necessary to optimize the benefits of day-case surgery for both the patient and the health care provider. However, outcome studies are needed to evaluate the effects of these newer therapeutic approaches with respect to postoperative side effects, cost and important recovery variables. Recent studies suggest that factors other than pain per se must be controlled in order to reduce postoperative morbidity and facilitate the recovery process. Not surprisingly, the anaesthetic technique can influence the analgesic requirements and the likelihood of emesis in the early postoperative period. Although opioid analgesics will continue to play an important role, the adjunctive use of both local anaesthetic agents and NSAIDs will probably assume an even greater role in the future. Use of drug combinations (e.g., opioids with local anaesthetics, $\mathrm{alpha}_{2}$ agonists and/or NSAIDs) may provide for improved analgesia with fewer opioid-related side effects than narcotic analgesics alone. Finally, safer and simpler analgesic delivery systems are needed to improve our ability to provide cost-effective pain relief after day-case surgery in the future.

In conclusion, as a result of our enhanced understanding of the mechanisms of acute pain and the physiological basis of nociception, the provision of "stress free" anaesthesia with minimal postoperative discomfort is now possible for most patients undergoing ambulatory surgical procedures. The aim of any analgesic technique should not only be to lower the pain scores but also to facilitate earlier mobilization and to reduce perioperative complications, in particular PONV. In future, clinicians should be able to effectively treat postoperative pain using a combination of "balanced," "preemptive," and "peripheral" analgesia techniques without producing emetic sequelae.

\section{References}

1 Armitage E. Postoperative pain-prevention or relief (Editorial). Br J Anaesth 1989; 63: 136-8.

2 Kehlet H, Dahl JB. The value of "multimodal" or "balanced analgesia" in postoperative pain treatment. Anesth Analg 1993; 77: 1048-56.

3 Souter AJ, Fredman B, White PF. Controversies in the perioperative use of nonsteroidal antiinflammatory drugs. Anesth Analg 1994; 79: 1178-90.

4 Watcha MF, White PF. Postoperative nausea and vomiting: its etiology, treatment and prevention. Anesthesiology 1992; 77: 162-84.

5 Kapur PA. The big "little problem" (Editorial). Anesth Analg 1991; 73: 243-5.

6 Smith I, White PF, Nathanson M, Gouldson $R$. Propofol: an update on its clinical uses. Anesthesiology 1994; 81: $1005-43$. 